\title{
A Review on Systemic and New Cancer Hallmarks
}

\section{Akruti Amol Ingole ${ }^{1}$, Anjali Vijay Petle ${ }^{2}$, Surbhi Balwant Dhoke ${ }^{3}$, Piyush Jagdish Balgote ${ }^{4}$, Anirban Goutam Mukherjee ${ }^{5}$ and Uddesh Ramesh Wanjari $^{3 *}$}

${ }^{1}$ Department of Biotechnology, Priyadarshini Institute of Engineering and Technology, Nagpur, Maharashtra, India

${ }^{2}$ Department of Biochemistry, RTM Nagpur University, Nagpur, Maharashtra, India ${ }^{3}$ Department of Biochemistry, Kamla Nehru Mahavidyalaya, Nagpur, Maharashtra, India

${ }^{4}$ Molecular Biology and Genetic Engineering, RTM Nagpur University, Nagpur, Maharashtra, India

${ }^{5}$ Department of Biosciences, School of Bio-Sciences and Technology, Vellore

Institute of Technology, Vellore, Tamil Nadu, India

*Corresponding Author: Uddesh Ramesh Wanjari, Department of Biochemistry, Kamla Nehru Mahavidyalaya, Nagpur, Maharashtra, India.
Received: August 13, 2021

Published: August 19, 2021

(C) All rights are reserved by Uddesh Ramesh Wanjari., et al.

\section{Abstract}

The body system is dependent on each other through communication, cancer development can be supported by the new cancerinduced pathological systemic networks (CISPN), and the entire organism is "cancerized." The significant anomaly in the transition of epithelial cells to mesenchymal cells plays a vital role in neural crest migration. The metastatic program follows a transformed cellular communication process that, activated once, causes dispersed tumors at a distance from the original site. This review aims to provide an idea of various systemic and new hallmarks and several altered networking processes that eventually end up causing cancer. Keywords: Cancer; Hallmarks; Inflammation; Cytokines; Tumor

\section{Introduction}

Cancer is a cluster of cells that divide, capture, and spread anywhere in an indiscriminate manner. There is a crucial need to detect the cellular and local stromal views and expand the standpoint by considering the entire organism. Topographically distinguished cancer cells can interact by developing a cascade that communicates with the organism through cancer-induced systemic pathogenic networks. In this paper, the systemic hallmarks of cancer appear to respond to this communication [1].

According to systemic biology, organisms are multiplex, nexus, sub-layered communication networks. The networks consist of metabolic intermediates, miRNA, and signaling factors, i.e. proteins, lipids, ions, and the networks can also be the interactions between the different cell types. The network involves different body systems interactions like endocrine, nervous, and immune systems. These networks are co-dependent and interplay by interacting and influencing each other $[1,2]$.

Cancer is a multiplex disease with a significantly higher degree of communication networks at various levels (i.e. organismic and cellular). The active entity of several communicating components functions and shows the dependency combined [53]. Macroscopi- 
cally, experimentally derived data assembled over more than a decade encourage the concept of the cancer system developed by the geographically differentiated cancer tissues (the primary tumor, the local, and the distant metastasis) $[1,3]$.

There are several factors listed with their systemic effects generated by the tumors, i.e., interleukin IL-8, stromal cell-derived factor-1(SDF-1), platelet-derived growth factor (PDGF), vascular endothelial growth factor (VEGF), fibroblast growth factor, growthrelated oncogene- $\alpha$ (CXCL1), lectin, angiogenin, normal T-cell expressed (CCL5), sonic hedgehog homolog, and osteopontin. The systemic standpoint should be indefinite up to stage IV cancers clinically. When the tumors are localized in many cases, and systemic effects appear, a notable proportion of cancers is incurable $[1,4]$.

\section{Cancer as a developmental disease}

Genes of organisms play a critical role in cancer and developmental stages (embryogenesis). It is the point that the embryo lacks the functional circulatory system, and therefore, in the metastatic process, the developmental processes are different. Some genes (i.e., Hedgehog, TGF- $\beta$, Nuclear hormone receptors, Notch, Wnt) are involved in the progression and the maintenance of multicellularity dysregulated in the stem cells and metastasis $[5,7]$.

Cancer cells regulate and forth between various cascades and may show a regulative cellular stage; it will cause the formation of a non-neoplastic phenotype [49]. In most cases, the metastatic process is due to epithelial to mesenchymal cell transition; it produces the factors such as HGF, Wnt, TGF- $\beta$, tumor necrosis factor (TNF)- $\alpha$, and PDGF by the activated tumor cells [6]. The surrounding tumor stroma central regulators of developmental stages (embryogenesis) such as Snail, Zeb1, transcription factor Twist, Zeb2 are considered the epithelial to mesenchymal transition (ETM) central regulators that drive metastasis. These programs may be propagated by miRNAs that controlled various genes simultaneously $[1,7]$.

The division, invasion, and metastasis processes may be separate and different in various types of cancer [50,51]. These steps may describe the certainty that cancers developing in different tissues have different tendencies to increase in size (locally), metastasize, and invade the encircling stroma, indicating that cancer stem cells (CSCs) are a minute population of cells in the tumors that show the capability of differentiation, tumorigenicity, and selfrenewable when transferred into an animal host [8].

The primary tumor-metastasis network

Clinically has been observed, the distal metastases and the primary tumor are co-dependent and interlinked. For example, in some renal cell cancer instances, exterminating the primary tumor has reverted to distal metastasis [9]. The niche's six pre-metastatic characteristics, namely vascular permeability, inflammation, organotropism, immunosuppression, angiogenesis, reprogramming, and lymphangiogenesis, promote the niche to support metastasis and tumor cell colonization. The soluble molecules secreted by the tumors responsible for developing and forming a niche are placental growth factor, granulocyte colony-stimulating factor (G-CSF), and vascular endothelial growth factor receptor- $\alpha$ (VEGF- $\alpha$ ) [10].

Exosomes are responsible for the composition of the premetastatic niche and the interaction between bone marrow, distal metastasis site, and the tumor. With the aid of the exosomes, DNA, miRNAs, mRNAs, and proteins are transported functionally between various types of cells, promoting the activities (biological) of non-tumor and tumor cells and promoting tumor growth, metastasis, drug resistance, invasion, and angiogenesis [11].

\section{The network of systemic inflammation}

The well-known connection between cancer development, local stromal inflammation, and the molecular cascades in charge of this connection has been recognized. As any physician would certify, inflammation is an essential criterion in the characteristic of cancer development, including the tumor cell's capability to metastasize [1]. Grivennikov., et al. explained that various types of inflammation induce progression and development of cancer, distinguished by intensity, mechanism, result, and causes. Chronic inflammation is broadly connected with autoimmune diseases or other infections; prolonged exposure to obesity and environmental irritants causes inflammation [12].

Transcription of inflammation-related genes is stimulated by DNA methylation, involving chemokine receptor 4 (CXCR4) and serum amyloid A (SAA) in modified clear cell carcinoma. This event is followed by tumor-producing cancer cell-intrinsic inflammation through epigenetic remodeling. This innovative concept shows an epigenetic foundation for the identified hallmarks of cancer gives 
compelling proof of the essential nature of epigenetic dysfunction [13].

\section{The global metabolism/cachexia network}

Cancer cells have higher metabolic maintenance rates; needed a massive count of energy to help their metabolism. The complete metabolic separation of cancer tissues demonstrates high aerobic glycolysis, increased glutaminolysis flux, elevated lipid, and amino acid metabolism. There is a reverse conversion of lactate to glucose in the liver cells (Oncogenic Cori Cycle); instead of glucose and lactate, there is also a need for other nutrients such as glutamine, glycine, and aspartate synthesize purines and pyrimidine $[14,15]$. Serine is needed to synthesize membrane lipid components, branched amino acids, lipids, and acetate. Stromal mitophagy, catabolism, and autophagy occur to fuel tumor anabolic growth, inducing metastasis and progression [1].

The multiple organ syndrome cachexias cause alterations in organs or tissues besides the tumor itself and the other organs, including the muscle cells, adipose tissue, pancreas, and liver cells (hepatocytes). It causes up-regulation of impaired anabolism, tumor-forming inflammatory cytokines secretion, and neuroendocrine anomalies. Cachexia is very high among cancer functions, mainly in gastric and pancreatic cancer [16].

Insulin and glucagon are secreted by the pancreas and play a crucial role in cachexia. The increase in the level of insulin resistance can cause cancer growth and is involved in cancer progression. During cancer development, a higher secretion of glucagon in alpha islet cells of the pancreas may also stimulate liver gluconeogenesis. Insulin, catecholamines, and atrial natriuretic peptides can cause the degradation of lipid $[17,18]$.

Similarly, structural and functional heart changes that cause cardiac failure are also involved in cachexia syndrome. In cardiac cachexia (in terms of cancer), there is skeletal muscle and function loss. Some patients with cachexia also suffer from remodeling, dysfunction, and cardiac atrophy $[1,15]$.

\section{The thrombosis network}

The various blood components and the lymphatic system in metastasis have gained popularity in cancer-targeted therapies for blood components and other lymphatic factors to treat cancer. Cancer and thrombosis exhibit reverse communication between them; on the other side, cancer cells causing the formation of clots, and the synthesis of clotting proteins initiate the growth of cancer and circulation [19]. Hypercoagulability is a cancer condition that removes procoagulant factors, such as tissue factor (TF). Crucial TFs carry out Cancer-associated coagulopathy and thromboembolic disorders. Cancer cell's communications with the neutrophils and platelets donate to extravasation, cancer adhesion, and the initiation of metastasis lesions [20].

\section{The neuro-endocrine network}

In some circumstances, the peripheral system secret the signals against systemic inflammation, and the hypothalamus combine those signals and then translates them into neuroendocrine perturbations, global metabolic derangements, and changed neuronal signaling [21]. The cytokines, IL-1 $\beta$ and TNF- $\alpha$, are secreted in the periphery during cancer development, and their modification occurs in the hypothalamus [21,22].

The different barriers display challenges to preventing excessive cell proliferation and cancer development, causing the intimidating companion of cancer. Tumor cells only modify the existing cellular and molecular mechanisms but avoid the pathways that do not allow tumor formation [23]. Tumor-producing cells that develop through differentiation have also been identified in intestinal tumors. Increased NF- $\kappa B$ signaling causes the stabilization of $\beta$-catenin to activate $\beta$-catenin/TCF transcription, with tumorforming stem-like properties developing dedifferentiation of nonstem intestinal epithelial cells to intestinal epithelial cells $[24,25]$.

\section{Dedifferentiation and transdifferentiation}

In the genome of a differentiated adult cell, there is information to transform into any cell type. Cancer cells have the potential to dedifferentiate by abducting four Yamanaka factors: c-MYC, Kruppel-like factor 4 (KLF4), Sex-determining region Y-box 2 (Sox2), and Octamer-binding transcription factor 3/4 (Oct-3/4). The cancer is immortalized, recurring, and resistant to therapy when non-CSC cells are dedifferentiated into CSCs [26]. Yamanaka factors' capacity to dedifferentiate plays a vital role in a variety of malignancies. Oct4 suppresses progenitor cell development and increases the number of undifferentiated cells, which is observed in seminomas. Ewing's sarcoma, breast cancer, and brain cancer have Sox2, whereas colon cancer has KLF4. Misrepresentation of MYC has been linked to several cancers. Yamanaka factors contributed to the development of targeted therapy [31]. 
Cancer stimulates the Waddington landscape allowing cells to select their destination, and they can become fundamental through dedifferentiation even at their final state of differentiation. Following the loss of APC in acute promyelocytic leukemia (APML), oncogenic KRAS mutations develop, resulting in tumorigenesis [1]. PML-RAR fusion gene expression prevents granulocyte differentiation and remains cancer cells in the promyelocytic precursor, while all-trans-retinoic can stimulate progenitor cells to differentiate into granulocytes [32].

\section{The cell fate in pancreatic cancer}

In pancreatic adenocarcinoma, petite GTPase KRAS-activating mutants have been found in 90 percent of pancreatic cancers. KRAS and MYC cause cancer malignancy by transforming different cells into stem cells. MYC promotes metastatic cell regeneration and cancer potentiation. The initiating mechanism is a synergistic interaction of active SOX9 and KRAS factors that lead to pancreatic acinar dedifferentiation in the form of the phenotype of the duct and intraepithelial neoplasia [1,27].

Therapy resistance via lineage plasticity through dedifferentiation

Dedifferentiation is a hallmark of immune system evasion and has been related to treatment-resistant melanoma. Decreased microphthalmia-associated transcription factor (MITF) leads to resistance to BRAF inhibition, which leads to an increase in the tyrosine kinase AXL receptor, PDGFR, and EGFR [28].

Dedifferentiation can also be an essential therapy objective, such as ferroptosis, iron-dependent cell death in melanoma that produces synthetic lethality when coupled with other immunotherapeutic medicines. Dedifferentiation-related changes have been found in melanoma patients as early as the first week of therapy; prostate and breast cell cancers benefit from it. Loss of p53 and Rb reductants causes reversal of prostate cancer by re-regulation of the Sox 2 transcription factor, resulting in cells converting to the endocrine neurotype phenotype [29]. The Wnt/-catenin signal triggered by physical stress and tissue pressure produced by adenocarcinoma also degrades fat into myofibroblasts in breast cancer [30].

Dedifferentiation is a telltale symptom of cancer. During dedifferentiation, cancer cells can be targeted by combining treatments to prevent early resistance to therapeutics due to lineage plasticity conferred [1,33], developing the state of permanent differentiation to combat dedifferentiation [1,33], and differentiation of undifferentiated cells into a safe cell line, for example, differentiated breast tumors to fatten $[1,33]$.

\section{Epigenetic dysregulation}

In melanoma formation, differentiation into progenitor cells is speed-limited but is supported by epigenetic machinery. Due to the state of chromatin and its modifying enzymes, Epigenetics regulates the clock's return to a pluripotent state and increases DNA demethylation by using vitamin-C [34]. Loss of DNA methylation was detected in a large portion of tumor cells by Vogelstein and Feinberg. According to them, the activation of cancer could lead to hypomethylation of $\mathrm{CpG}$ islets. According to the Holliday definition, epigenetics is a change in phenotype without a change in genotype due to a change in gene expression without a change in DNA [36].

\section{Sustained proliferative signaling}

Mutations in isocitrate dehydrogenase (IDH) function are seen in many tumors. It shifts DNA binding protein affinity to CTCF (CCCTC binding agent), which is far more sensitive than methylation conditions. A dominant oncogene among gliomas is plateletderived growth factor receptor-A (PDGFR-A), which reduces epigenetic regulation disorder in CTCF insulation. In addition to glioma, this mechanism is also seen in the endometrium, esophagus, gastric, and large intestine tumors [1,37].

\section{Evading growth suppressors}

Following the coding of p16INK4a, the retinoblastoma (Rb) protein is kept hypophosphorylated, resulting in the development of the $\mathrm{Rb} / \mathrm{E} 2 \mathrm{~F}$ suppressor complex, which inhibits the growth and G1 phase of the cell division cycle. Tumor suppressors, such as p16INK4a, are epigenetically silenced and impair growth through promoter hypermethylation. Increasing the Zeste homolog 2 (EZH2) regenerator escapes excess growth pressure by suppressing CDKN2A [38].

\section{Metastasis}

Epigenetic suppression of CDH1, which encodes E-cadherin, is accomplished by employing the snail transcription factor EMT to the CDH1 promoter and suppressing H3K27me3 [1,39]. 


\section{Replicative immortality}

A pathway based on telomerase-independent homologous recombination is alternative telomere lengthening (ALT). Tumor cells use ALT to maintain telomere length and overcome the hayflick limit. Interference between epigenetics and genetic mutations causes the histone H3.3 variant in the telomerase and heterochromatic state to be disrupted, and ALT facilitated [40].

\section{Angiogenesis induction}

Epigenetics plays a crucial role in angiogenesis by increasing hypoxia-1 induction and vascular endothelial growth factors (VEGF), decreasing von Hippel - Lindau (VHL), and reducing the effectivity of p53. Choriocarcinoma is a trophoblast-derived vascular tumor that induces the epigenetic silence of FLT1 through hypermethylation, thus inhibiting the soluble Fms-like tyrosine kinase-1 (sFLT1), which is anti-angiogenic [41].

\section{Bypassing cell death}

Glioblastoma multiforme is invasive cancer with a poor prognosis. Binding the apoptosis-inducing ligand TRAIL to the human death receptor 4 (DR4) is one suggested therapy. Epigenetic silence reduces promoter TRAIL/DR4-mediated apoptosis through promoter methylation. CXCL14 is an apoptotic chemokine that is an option for recurrent epigenetic silencing lung adenocarcinomas [42].

\section{Immune evasion}

Epigenetics is essential for immune system function by delivering MHC class-1 antigen to T + CD8 cells. The transactivator NLRC5 controls MHC class-I transcription, but the promoter in the NLRC5 area is methylated during cancer, and eventually the expression of MHC class-I is reduced [43].

\section{Deregulating cellular energetics}

Cancer cells can employ glycolysis for glucose metabolism under aerobic circumstances instead of oxidative phosphorylation, including the PI3K/AKT/mTOR pathway and MYC and HIF-1 signaling [44]. PTEN, VHL, LKB1, and prolyl hydroxylases are tumor suppressors in this pathway extinguished by promoter hypermethylation [1].

\section{Genomic instability and mutation}

Transposable elements (TE) are several duplicate genomes that tend to impair the genome stability of adjacent genes. These genes are typically silenced, but during cancer, their extinction regulation is lost [36].

\section{Altered microbiome}

Helicobacter pylori cause 90 percent of stomach malignancies. The cytotoxin genes vacA and CagA are linked with the presence of $H$. pylori. CagA disrupts the mitotic spindle checkpoint, resulting in chromosomal instability $[1,46,52]$. MLH1 is an essential DNA repair gene. The hypermethylation of MLH1 is caused by an increase in DNA methyltransferases (DNMTs). Mutations in Colibactin can cause tumors to develop. Paget's seed (cancer cells) and soil (tumor microenvironment) hypotheses are related to microbiota carcinogenesis [46].

\section{Altered neuronal signalling}

$\beta$-blockers for inhibiting tumor progression

Chronic stress is a risk factor for cancer. Noradrenaline secreted by the sympathetic nerves increases its circulation during chronic stress, and $\beta$-adrenergic receptors mediate its pro-tumorigenic effects. For example, angiogenesis following the release of noradrenaline and its effect on $\beta$-adrenergic receptors in prostate cancer in mice [47].

\section{Nerves and the tumor microenvironment}

The 2-adrenergic receptor can allow tumors to escape the immune system by decreasing lymphocyte outflow and lowering $\mathrm{T}$ cell absorption [30,47]. During the studies, breast cancer progression increased rapidly by manipulating the autonomic nerves, but local sympathetic denervation slowed tumor growth and decreased checkpoint markers (e.g., FOXP3, PD-L1, and PD-1) that drive immune suppression. It is characterized by nerve tumors and signals that interact with their tiny surroundings, leading to cancer development and metastasis [48].

\section{Conclusion}

Dedifferentiation/transdifferentiation and epigenetic dysregulation are the significant factors behind cancer hallmarks, while altered microbiome and changed neuronal signaling are the two activating factors $[1,46]$. Disruption of epigenetics provides an opportunity for cancer to develop. The microbiome should be used to increase the outcome of treatment. Altering neural signals causes angiogenesis to proliferate and metastasize [12,22,23]. Stimulating oncogene in cancer cells results in pro-inflammatory transcription factors appearing within tumor cells, i.e., signal transducer, the ac- 
tivators of transcription (STAT3 or HIF-1 $\alpha$ ), and nuclear factor-kappa B (NF- $\kappa$ B) [24]. The initial systemic hallmarks include the cancer system that links the bone marrow, the distal metastasis, and global inflammation. Immunity inhibition, global inflammation, the metabolic changes causing cachexia, the tendency to thrombosis, and neuroendocrine changes comprise the major systemic hallmarks of cancer [1]. More research is required to understand every systemic hallmark effectively so that innovative ways can be developed to target various processes and effectively control cancer.

\section{Bibliography}

1. Paul D. "The systemic hallmarks of cancer". Journal of Cancer Metastasis and Treatment 6 (2020).

2. Zhong Z., et al. "Wnts and the hallmarks of cancer". Cancer and Metastasis Reviews 39.3 (2020): 625-645.

3. Senga SS and Grose RP. "Hallmarks of cancer-the new testament”. Open Biology 11.1 (2021): 200358.

4. Hong WX., et al. "Intratumoral immunotherapy for early-stage solid tumors”. Clinical Cancer Research 26.13 (2020): 30913099.

5. McAllister SS and Weinberg RA. "The tumor-induced systemic environment as a critical regulator of cancer progression and metastasis". Nature Cell Biology 16.8 (2014): 717-727.

6. Dudás J., et al. "Epithelial to mesenchymal transition: A mechanism that fuels cancer radio/chemoresistance". Cells 9.2 (2020): 428.

7. Vincent MD. "The animal within: carcinogenesis and the clonal evolution of cancer cells are speciation events sensu stricto". Evolution 64.4 (2010): 1173-1183.

8. Gooding AJ and Schiemann WP. "Epithelial-mesenchymal transition programs and cancer stem cell phenotypes: mediators of breast cancer therapy resistance". Molecular Cancer Research 18.9 (2020): 1257-1270.

9. Flanigan RC and Yonover PM. "The role of radical nephrectomy in metastatic renal cell carcinoma". Seminar on Urology and Oncology 19.2 (2001): 98-102.
10. Kaplan RN., et al. "VEGFR1-positive haematopoietic bone marrow progenitors initiate the pre-metastatic niche". Nature 438.7069 (2005): 820-827.

11. Guo L and Guo N. "Exosomes: Potent regulators of tumor malignancy and potential bio-tools in clinical application". Critical Reviews in Oncology/Hematology 95.3 (2015): 346-358.

12. Grivennikov SI., et al. "Immunity, inflammation, and cancer". Cell 140.6 (2010): 883-899.

13. Nishida J., et al. "Epigenetic remodelling shapes inflammatory renal cancer and neutrophil-dependent metastasis". Nature Cell Biology 22.4 (2020): 465-475.

14. Tisdale MJ. "Mechanisms of cancer cachexia". Physiology Review 89.2 (2009): 381-410.

15. Fearon KC., et al. "Cancer cachexia: mediators, signaling, and metabolic pathways". Cell Metabolism 16.2 (2012): 153-166.

16. Porporato PE. "Understanding cachexia as a cancer metabolism syndrome". Oncogenesis 5.2 (2016): e200.

17. Wang F., et al. "The Warburg effect in human pancreatic cancer cells triggers cachexia in athymic mice carrying the cancer cells". BMC Cancer 18.1 (2018): 1-12.

18. Chasen M., et al. "Immunomodulatory agents for the treatment of cachexia". Current Opinion in Supportive and Palliative Care 8.4 (2014): 328-333.

19. Falanga A., et al. "Mechanisms and risk factors of thrombosis in cancer". Critical Reviews in Oncology/Hematology 118 (2017): 79-83.

20. Versteeg HH., et al. "Inhibition of tissue factor signaling suppresses tumor growth”. Blood 111.1 (2008): 190-199.

21. Jiang SH., et al. "Systemic Regulation of Cancer Development by Neuro-Endocrine-Immune Signaling Network at Multiple Levels". Frontiers in Cell and Developmental Biology 8 (2020): 1056.

22. Braun TP., et al. "Central nervous system inflammation induces muscle atrophy via activation of the hypothalamic-pituitaryadrenal axis". Journal of Experimental Medicine 208.12 (2011): 2449-2463. 
23. Yehya AHS., et al. "Angiogenesis: managing the culprits behind tumorigenesis and metastasis". Medicina 54.1 (2018): 8.

24. Wang L., et al. "Fbxw11 promotes the proliferation of lymphocytic leukemia cells through the concomitant activation of NF- $\mathrm{B}$ and $\beta$-catenin/TCF signaling pathways". Cell Death and Disease 9.4 (2018): 1-12.

25. de Souza CF., et al. "A distinct DNA methylation shift in a subset of glioma CpG island methylator phenotypes during tumor recurrence". Cell Reports 23.2 (2018): 637-651.

26. Takahashi $\mathrm{K}$ and Yamanaka S. "Induction of pluripotent stem cells from mouse embryonic and adult fibroblast cultures by defined factors". Cell 126 (2006): 663-676.

27. Ischenko., et al. "Direct reprogramming by oncogenic Ras and Myc". Proceedings of the National Academy of Sciences of the United States of America 110.10 (2013): 3937-3942.

28. Müller J., et al. "Low MITF/AXL ratio predicts early resistance to multiple targeted drugs in melanoma". Nature Communication 5 (2014): 5712.

29. Mu P., et al. "SOX2 promotes lineage plasticity and antiandrogen resistance in TP53- and RB1-deficient prostate cancer". Science 355.6320 (2017): 84-88.

30. Li Y., et al. "Compression-induced dedifferentiation of adipocytes promotes tumor progression". Science Advances 6.4 (2020): eaax5611.

31. Halim S., et al. "Analysis of cell proliferation and tissue remodelling uncovers a KLF4 activity score associated with poor prognosis in colorectal cancer". British Journal of Cancer 119.7 (2018): 855-863.

32. Strickland S., et al. "The induction of differentiation in teratocarcinoma stem cells by retinoic acid". Cell 15.2 (1978): 393403.

33. Augimeri G., et al. "Natural and synthetic PPAR $\gamma$ ligands in tumor microenvironment: a new potential strategy against breast cancer". International Journal of Molecular Sciences 21.24 (2020): 9721.

34. Chen J., et al. "H3K9 methylation is a barrier during somatic cell reprogramming into iPSCs". Nature Genetics 45.1 (2013): $34-42$.
35. Giorda R. "Principles of epigenetics and DNA methylation". In Developmental Human Behavioral Epigenetics (2020): 3-26.

36. Zhang W., et al. "Global DNA hypomethylation in epithelial ovarian cancer: passive demethylation and association with genomic instability". Cancers 12.3 (2020): 764.

37. Jankowska S., et al. "Molecular classification of glioblastoma based on immunohistochemical expression of EGFR, PDGFRA, NF1, IDH1, p53 and PTEN proteins". Polish Journal of Pathology 72.1 (2021): 1-10.

38. Kim KH and Roberts CW. "Targeting EZH2 in cancer". Nature Medicine 22.2 (2016): 128-134.

39. Sun J., et al. "N-terminal truncated carboxypeptidase E represses E-cadherin expression in lung cancer by stabilizing the Snail-HDAC complex". American Journal of Cancer Research 10.3 (2020): 925.

40. Jain SU., et al. "Histone H3. 3 G34 mutations promote aberrant PRC2 activity and drive tumor progression". Proceedings of the National Academy of Sciences 117.44 (2020): 27354-27364.

41. Sasagawa T., et al. "Production of an anti-angiogenic factor sFLT1 is suppressed via promoter hypermethylation of FLT1 gene in choriocarcinoma cells". BMC Cancer 20.1 (2020): 112.

42. Shabgah AG., et al. "Chemokine CXCL14; a double-edged sword in cancer development". International Immunopharmacology 97 (2021): 107681.

43. Yoshihama S., et al. "NLRC5/MHC class I transactivator is a target for immune evasion in cancer". Proceedings of the National Academy of Sciences of the United States of America 113.21 (2016): 5999-6004.

44. Cirone M. "Cancer cells dysregulate PI3K/AKT/mTOR pathway activation to ensure their survival and proliferation: mimicking them is a smart strategy of gammaherpesviruses". Critical Reviews in Biochemistry and Molecular Biology (2021): 1-10.

45. Nishida J., et al. "Epigenetic remodelling shapes inflammatory renal cancer and neutrophil-dependent metastasis". Nature Cell Biology 22.4 (2020): 465-475.

46. Hatakeyama M. "Helicobacter pylori CagA and gastric cancer: a paradigm for hit-and-run carcinogenesis". Cell Host Microbe 15.3 (2014): 306-316. 
47. Partecke LI., et al. "Chronic stress increases experimental pancreatic cancer growth, reduces survival and can be antagonised by beta-adrenergic receptor blockade". Pancreatology 16.3 (2016): 423-33.

48. Kamiya A., et al. "Genetic manipulation of autonomic nerve fiber innervation and activity and its effect on breast cancer progression". Nature Neuroscience 22.8 (2019): 1289-1305.

49. Mukherjee AG., et al. "A Review on The Usefulness of Various Eukaryotic Pigments and Metabolites in Cancer Treatment". WJPR 9.10 (2020): 587-611.

50. Mukherjee AG., et al. "A Review on the Present and Future Aspects of Various Prokaryotic Pigments and Metabolites Demonstrating Anti-Cancerous Properties". International Journal of Engineering Research and Technology 9.7 (2020): 1262-1266.

51. Ingole AA., et al. "A Review on Carcinogenic Heavy Metals". International Journal of Engineering Research and Technology 10.3 (2021): 403-411.

52. Mukherjee AG and Wanjari U. "A Short Review on Various Aspects of Gene Sequencing and Metagenomics to Study Human Gut Microbiome". International Research Journal of Engineering and Technology 7.7 (2020): 1280-1284.

53. Mukherjee AG., et al. "The CRISPR-Cas System as a Technology to Redefine Industrial Biotechnology". Acta Scientific Microbiology 4.6 (2021): 45-52.

\section{Volume 5 Issue 9 September 2021}

(C) All rights are reserved by Uddesh Ramesh Wanjari., et al. 